\title{
Sub-milliarcsecond imaging of a bright flare and ejection event in the extragalactic jet $3 \mathrm{C} 111^{\star}$
}

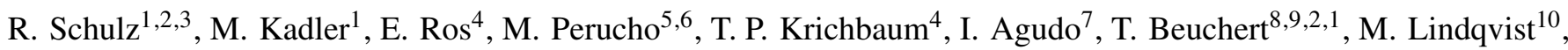 \\ K. Mannheim ${ }^{1}$, J. Wilms ${ }^{2}$, and J. A. Zensus ${ }^{4}$
}

\author{
${ }^{1}$ Lehrstuhl für Astronomie, Universität Würzburg, Campus Hubland Nord, Emil-Fischer-Strasse 31, 97074 Würzburg, Germany \\ e-mail: matthias.kadler@astro.uni-wuerzburg.de \\ 2 Dr. Remeis Sternwarte \& ECAP, Universität Erlangen-Nürnberg, Sternwartstr. 7, 96049 Bamberg, Germany \\ 3 Netherlands Institute for Radio Astronomy (ASTRON), Oude Hoogeveensedijk 4, 7991 PD Dwingeloo, The Netherlands \\ e-mail: r.f.schulz@issc.leidenuniv.nl \\ 4 Max-Planck-Institut für Radioastronomie, Auf dem Hügel 69, 53121 Bonn, Germany \\ 5 Departament d'Astronomia i Astrofísica, Universitat de València, 46100 Burjassot, València, Spain \\ 6 Observatori Astronòmic, Universitat de València, Parc Científic, C. Catedrático José Beltrán 2, 46980 Paterna, València, Spain \\ 7 Instituto de Astrofísica de Andalucía, CSIC, Apartado 3004, 18080 Granada, Spain \\ 8 European Southern Observatory, Karl-Schwarzschild-Str. 2, 85748 Garching bei München, Germany \\ 9 Anton Pannekoek Institute for Astronomy, PO Box 94249, 1090 GE Amsterdam, The Netherlands \\ 10 Department of Earth and Space Sciences, Chalmers University of Technology, 43992 Onsala, Sweden
}

Received 14 February 2020 / Accepted 4 August 2020

\begin{abstract}
Context. Flares in radio-loud active galactic nuclei are thought to be associated with the injection of fresh plasma into the compact jet base. Such flares are usually strongest and appear earlier at shorter radio wavelengths. Hence, very long baseline interferometry (VLBI) at millimeter(mm)-wavelengths is the best-suited technique for studying the earliest structural changes of compact jets associated with emission flares.

Aims. We study the morphological changes of the parsec-scale jet in the nearby $(z=0.049) \gamma$-ray bright radio galaxy $3 \mathrm{C} 111$ following a flare that developed into a major radio outburst in 2007.

Methods. We analyse three successive observations of 3C 111 at $86 \mathrm{GHz}$ with the Global mm-VLBI Array (GMVA) between 2007 and 2008 which yield a very high angular resolution of $\sim 45 \mu$ as. In addition, we make use of single-dish radio flux density measurements from the F-GAMMA and POLAMI programmes, archival single-dish and VLBI data.

Results. We resolve the flare into multiple plasma components with a distinct morphology resembling a bend in an otherwise remarkably straight jet. The flare-associated features move with apparent velocities of $\sim 4.0 \mathrm{c}$ to $\sim 4.5 \mathrm{c}$ and can be traced also at lower frequencies in later epochs. Near the base of the jet, we find two bright features with high brightness temperatures up to $\sim 10^{11} \mathrm{~K}$, which we associate with the core and a stationary feature in the jet.

Conclusions. The flare led to multiple new jet components indicative of a dynamic modulation during the ejection. We interpret the bend-like feature as a direct result of the outburst which makes it possible to trace the transverse structure of the jet. In this scenario, the components follow different paths in the jet stream consistent with expectations for a spine-sheath structure, which is not seen during intermediate levels of activity. The possibility of coordinated multiwavelength observations during a future bright radio flare in 3C 111 makes this source an excellent target for probing the radio- $\gamma$-ray connection.
\end{abstract}

Key words. galaxies: active - galaxies: jets - galaxies: individual: 3C 111 - techniques: high angular resolution

\section{Introduction}

A common signature of relativistic jets in radio-loud active galactic nuclei (AGN) is their strong variability in radio emission (e.g., Lähteenmäki \& Valtaoja 1999; Hovatta et al. 2008, 2009; Richards et al. 2014). This is generally explained by shocks traveling through the parsec-scale radio jet leading to the appearance of new features in high-resolution images on milliarcsecond (mas) scales (e.g., Marscher \& Gear 1985; Valtaoja et al. 1999; Savolainen et al. 2002; Fromm et al. 2013a). Moreover, combined studies of radio and $\gamma$-ray light curves reveal a correlation of flares occurring in both energy regimes with the

* The FITS-files of the GMVA images shown in Fig. 2 and Table A.4 are available at the CDS via anonymous ftp to cdsarc.u-strasbg. fr $(130.79 .128 .5)$ or via http://cdsarc.u-strasbg.fr/viz-bin/ cat $/ \mathrm{J} / \mathrm{A}+\mathrm{A} / 644 / \mathrm{A} 85$ radio emission often seen first at millimeter(mm) wavelengths (e.g., Lähteenmäki \& Valtaoja 2003; León-Tavares et al. 2011; Fuhrmann et al. 2014). In the $\mathrm{TeV} \gamma$-ray band, extreme variability on timescales as short as minutes has been observed (Albert et al. 2007; Aharonian et al. 2007; Aleksić et al. 2014), which suggests extremely small and compact substructure in AGN jets. Rapid broadband variability is not exclusive to blazars, and can also be seen in radio galaxies with small viewing angles of the jet to the line of sight (e.g., Jorstad et al. 2001; Marscher et al. 2010; Agudo et al. 2011; Schinzel et al. 2012; Karamanavis et al. 2016; Casadio et al. 2015). The favorable orientation and typically small distances allow us to probe substantially smaller linear scales in high-resolution imaging studies of radio-galaxy jets than in blazars (e.g., Boccardi et al. 2017).

An important open question concerns the physical origins of high-energy blazar variability. These are likely related to 
changes of particle acceleration and cooling processes in the most compact regions of the jets. Various propagation effects likely play a role but cannot be probed by any direct means due to the limited angular resolution at $\gamma$-ray energies. Frequent VLBI observations of $\gamma$-ray-bright radio galaxies at mm wavelengths as made possible with the Global mm-VLBI Array (GMVA ${ }^{1}$ ) in combination with single-dish flux density monitoring are the most appropriate method for performing such a study as they provide angular resolutions down to $\sim 40 \mu \mathrm{as}$, which can correspond to sub-parsec linear deprojected scales for nearby sources.

One prime target for such studies is the nearby radio galaxy $3 \mathrm{C} 111$ at a redshift $z=0.049$ (Véron-Cetty \& Véron 2010). The kiloparsec-scale radio morphology (Linfield \& Perley 1984) is consistent with the Fanaroff-Riley class II (Fanaroff \& Riley 1974). The parsec-scale jet exhibits apparent superluminal motion up to speeds of $\sim 8 c$ and the angle of the jet to the line of sight has been estimated to between $\sim 10^{\circ}$ and $\sim 20^{\circ}$ (although smaller angles have not been entirely excluded; Götz et al. 1987; Jorstad et al. 2005; Lewis et al. 2005; Kadler et al. 2008; Hogan et al. 2011; Lister et al. 2013; Beuchert et al. 2018). $3 \mathrm{C} 111$ has shown several radio flares in the past with two exceptional, long-lasting outbursts of several months above $10 \mathrm{Jy}$ in flux density in the $3 \mathrm{~mm}$ band (Alef et al. 1998; Trippe et al. 2011; Chatterjee et al. 2011). The first one occurred in early 1996 (Alef et al. 1998) and the second was observed in the middle of 2007 (Chatterjee et al. 2011). Both developed into complex features in the jet at $15 \mathrm{GHz}$ and $43 \mathrm{GHz}$ that differed from other components related to lower-activity phases (Kharb et al. 2003; Jorstad et al. 2005, 2017; Kadler et al. 2008; Chatterjee et al. 2011; Beuchert et al. 2018). 3C 111 has also been detected at $\gamma$-ray energies and seems to be a rather faint $\gamma$-ray emitter most of the time and becomes bright and detectable only during short flaring periods (Hartman et al. 2008; Grandi et al. 2012). It has been concluded that the site of the $\gamma$-ray flares of $3 \mathrm{C} 111$ is confined to a compact region smaller than $0.1 \mathrm{pc}$ inside the unresolved cm-VLBI core at distances of not more than $0.3 \mathrm{pc}$ from the central black hole.

This paper presents results of very high angular resolution observations with the GMVA over a period of one year during a major radio outburst. Schulz et al. (2013) presented preliminary images, but here we present the final images based on the fully calibrated data and subsequent results. The following section describes the radio data and their reduction (Sect. 2). Sections 3 and 4 present our results and a discussion of them, which is followed by a summary in Sect. 5 .

Throughout this paper, we adopt the $\Lambda \mathrm{CDM}$ cosmology with the parameters $H_{0}=70 \mathrm{~km} \mathrm{~s}^{-1} \mathrm{Mpc}^{-1}, \Omega_{\mathrm{M}}=0.3$, and $\Lambda=0.7$ (Freedman et al. 2001). Hence, at the redshift of 3C 111 we have a projected linear scale of 1 mas $\approx 0.96 \mathrm{pc}$. Assuming an inclination of the jet of $13^{\circ}$ (see above), the angular beam of the GMVA of (40-70) $\mu$ as can spatially resolve linear deprojected scales down to $\sim 0.17 \mathrm{pc}$. The linear resolution corresponds to about 9900 Schwarzschild radii assuming a mass of $1.8_{-0.4}^{+0.5} \times 10^{8} M_{\odot}$ for the central supermassive black hole as determined by Chatterjee et al. (2011).

\section{Observation and data reduction}

\subsection{GMVA observations}

Following the outburst of 3C 111 in 2007, we performed three successive observations with the GMVA at $86 \mathrm{GHz}$ on 2007

\footnotetext{
1 http://www.mpifr-bonn.mpg.de/div/vlbi/globalmm/
}

Oct. 15, 2008 May 11, and 2008 Oct. 14 (experiment code GK039A,B and GK040). The configuration of the GMVA at the time of our observations comprised five European and eight North-American telescopes, which are listed in Table A.1.

The GMVA data were correlated with the VLBI correlator at the Max Planck Institute for Radio Astronomy (MPIfR, Germany). We used the Astronomical Imaging Processing System (AIPS, Greisen 2003) to perform the a priori amplitude and phase calibration. For this purpose, we corrected for atmospheric attenuation at each station which is a critical issue for $\mathrm{mm}$-VLBI because of the increasing opacity of the atmosphere at high radio frequencies. In addition, measurements of the antenna's system temperature and gain factors were applied. The phases were calibrated using the global fringe-fitting algorithm (Schwab \& Cotton 1983).

The $86 \mathrm{GHz}$ GMVA data were further self-calibrated and imaged with the software package DIFMAP (Shepherd 1997). An initial model was created using the CLEAN algorithm (Högbom 1974) and phase self-calibration combined with flagging of bad data points which was followed by amplitude self-calibration over the whole observing time. This procedure was repeated with subsequent smaller time intervals for amplitude self-calibration. The images were produced using the final CLEAN-model convolved with the CLEAN-beam, which is a two-dimensional Gaussian approximation of the dirty beam, and adding the residual noise. The hybrid imaging process was performed with natural weighting of the visibilities. The properties of the resulting images are listed in Table 1.

We tested the absolute amplitude calibration by comparing the total flux density with quasi-simultaneous single-dish measurements from the F-GAMMA programme at $86 \mathrm{GHz}(2007$ Oct. 9: $12.06 \pm 0.22 \mathrm{Jy}, 2008$ May 2: $4.05 \pm 0.16 \mathrm{Jy}, 2008$ Oct. 7: $4.06 \pm 0.21 \mathrm{Jy})$. We consistently use F-GAMMA measurements (see Sect. 2.2) for our amplitude-calibration check and scaling, which benefit from simultaneous multifrequency measurements that reduce the risk of systematic gain offsets. We also tested the independent additional adjacent POLAMI measurements, which lead to consistent results within the F-GAMMA measurement uncertainties.

We found a significant difference between the single-dish and VLBI flux density for the first two observations by a factor of $1.5 \pm 0.2$ and $2.1 \pm 0.2$, respectively. It is unlikely that this discrepancy stems from missing flux density resolved out by VLBI because of the high observing frequency. In addition, the last observation is consistent with the single-dish measurements $(1.1 \pm 0.2)$. Hence, we consider uncertainties in the system temperature measurements and the gain curves of the telescopes to be the likely origin of the inconsistency between VLBI and single-dish flux density of the first two observations. We empirically corrected for this by scaling the CLEAN-model from the initial imaging by the factors given above. The new CLEAN-model was used to determine a constant amplitudecorrection factor for each telescope. The scaled visibilities were then imaged and self-calibrated again. For the initial images, we estimated an uncertainty of the total flux density of about $15 \%$ based on repeating the imaging process several times. This uncertainty applies to the scaled data and the resultant images. For the third GMVA observation, we estimated an additional systematic uncertainty of the flux density, because the $86 \mathrm{GHz}$ light curve suggests that this VLBI observation occurs during a rise in flux density. Based on an interpolation between the two adjacent F-GAMMA observations, we find that the flux density of the third observation might be underestimated by about $22 \%$. Because of uncertainty in the actual light-curve evolution, we 
Table 1. Properties of the $86 \mathrm{GHz}$ images.

\begin{tabular}{|c|c|c|c|c|c|c|c|c|}
\hline $\begin{array}{l}\text { Date }^{(a)} \\
\text { [YYYY-MM-DD] }\end{array}$ & $\begin{array}{l}\text { Date }^{(a)} \\
\text { [year] }\end{array}$ & GMVA configuration $^{(b)}$ & $\begin{array}{c}\sigma_{\text {noise }}{ }^{(b)} \\
{\left[\mathrm{mJy}^{-1} \text { beam }^{-1}\right]}\end{array}$ & $\begin{array}{c}S_{\text {peak }}(c) \\
{\left[\mathrm{Jy} \mathrm{beam}^{-1}\right]}\end{array}$ & $\begin{array}{c}S_{\text {total }}(d) \\
{[\mathrm{Jy}]}\end{array}$ & $\begin{array}{c}b_{\text {maj }}(e) \\
{[\mu \text { as }]}\end{array}$ & $\begin{array}{c}b_{\min }{ }^{(e)} \\
{[\mu \mathrm{as}]}\end{array}$ & $\begin{array}{c}b_{\mathrm{PA}}{ }^{(e)} \\
{\left[{ }^{\circ}\right]}\end{array}$ \\
\hline $2007-10-15$ & 2007.79 & Ef-On-Me-PdB-VLBA & 0.32 & $2.41 \pm 0.36$ & $11.8 \pm 1.8$ & 148 & 45 & -11 \\
\hline 2008-05-11 & 2008.36 & Ef-On-PV-VLBA & 0.16 & $0.93 \pm 0.14$ & $4.27 \pm 0.64$ & 152 & 59 & -16 \\
\hline $2008-10-14$ & 2008.78 & Ef-On-VLBA & 0.13 & $1.87 \pm 0.28^{(f)}$ & $3.78 \pm 0.57^{(f)}$ & 167 & 67 & -18 \\
\hline
\end{tabular}

Notes. ${ }^{(a)}$ Date of observation; ${ }^{(b)}$ GMVA elements with good data after calibration, imgaing and flagging: Effelsberg (Ef), Onsala (On), Metsähovi (Me), Plateau de Bure (PdB), Very Long Baseline Array (VLBA, the eight stations equipped with $86 \mathrm{GHz}$ receivers) image noise level; ${ }^{(c)}$ peak flux density of the image; ${ }^{\left({ }^{\prime}\right)}$ total flux density; ${ }^{(e)}$ major axis, minor axis, and position angle of the restoring beam. ${ }^{(f)}$ The flux density might be underestimated by about $22 \%$ (see Sect. 2.1).

prefer to use the scaling factors from real flux density measurements. Therefore, we did not scale the third VLBI observation as discussed above, but we indicate the systematic uncertainty for the third observation where necessary in this paper.

The self-calibrated data were modeled with two-dimensional circular Gaussian components using the fit routine implemented in DIFMAP. The aim of this process is to find a consistent description of the evolving brightness distribution of the jet throughout the three observations. The uncertainties on the parameters for each component were first calculated with a purely statistical approach based on Fomalont (1999) which may underestimate the true uncertainties because this approach does not account for systematic errors introduced during the aperture-synthesis processing, that is, by data editing, deconvolution, and (self-)calibration. Hence, we conservatively alter the uncertainties for the flux density of the components to the $15 \%$ level adopted from amplitude calibration. Similarly, we alter the uncertainties for the positions of the components to $50 \%$ of their major axis. The best-fit parameters of all components are listed in Table A.2.

\subsection{Ancillary data}

The F-GAMMA (Fermi-GST AGN Multi-frequency Monitoring Alliance) programme ${ }^{2}$ monitored a sample of $\gamma$-ray-bright blazars with the Effelsberg $100 \mathrm{~m}$ and the Pico Veleta $30 \mathrm{~m}$ radio telescopes over a broad range of radio frequencies from January 2007 till January 2015 (Angelakis et al. 2010, 2012, 2019; Fuhrmann et al. 2014, 2016). Here, we focus on measurements at $86 \mathrm{GHz}$ obtained with the Pico Veleta telescope between 2007 and 2009 (see Table A.4).

Additional flux density measurements of the source at $86 \mathrm{GHz}$ (see Table A.4), published in Agudo et al. (2014), are provided by the POLAMI (Polarimetric Monitoring of AGN at Millimeter Wavelengths) programme ${ }^{3}$ (Agudo et al. 2018a,b; Thum et al. 2018). The data were also taken with the IRAM $30 \mathrm{~m}$ telescope at the Pico Veleta Observatory in the same time frame as the F-GAMMA programme. The observing strategy and data reduction of the POLAMI programme is described in detail in Agudo et al. (2018a).

We further make use of selected VLBA data at $43 \mathrm{GHz}$ that are available in a fully calibrated state from the VLBA-BUBLAZAR monitoring programme ${ }^{4}$ (e.g., Marscher et al. 2011; Jorstad et al. 2017). These data were previously discussed by Chatterjee et al. (2011) and Jorstad et al. (2017). Similar to the

\footnotetext{
2 https://www3.mpifr-bonn.mpg.de/div/vlbi/fgamma/ fgamma.html

3 http://polami.iaa.es

4 https://www.bu.edu/blazars/VLBAproject.html
}

GMVA data, we fit two-dimensional Gaussian components to the visibilities to represent the brightness distribution of the jet. We selected observations which were close in time to the GMVA data. We also make use of previously published SMA flux density measurements at $230 \mathrm{GHz}$ over a similar time range to the F-GAMMA data (Chatterjee et al. 2011).

\section{Results}

\subsection{Single-dish light curve}

The $86 \mathrm{GHz}$ and $230 \mathrm{GHz}$ light curves between 01 July 2006 and 01 July 2009 are shown in the bottom panel of Fig. 1. The peak of the major outburst occurred around August 2007 and was followed by a smaller flare in late 2008 before returning to a more typical lower state.

The first GMVA observation was conducted only two months after the peak in the light curve at which time 3C 111 was clearly still in a very high state. The second GMVA observation was performed in a state when the single-dish light curve had already dropped to about half the peak value. The third GMVA observation took place close to the peak of the secondary smaller flare in late 2008 and the single-dish flux density measurement reached the same value as around the second GMVA epoch.

\subsection{Morphology and time evolution of the $86 \mathrm{GHz}$ jet}

Figure 2 shows the three images obtained by the GMVA observations. These are centered on the brightest feature and reveal a single-sided jet starting in the east-northeast direction. The first and second observations show an unusual change in the position angle of the jet at a distance of $\sim 0.5$ mas and $\sim 1$ mas, respectively. The jet seems to form a bend which increases in scale between both observations. By the time of the third observation the bend has evolved into a larger and more diffuse complex emission region. We also find faint diffuse emission at a distance of around 4 mas, which lies outside of the chosen plot range of Fig. 2 as it is not relevant for this study.

In order to better quantify the morphology of the jet and its changes over time, we use the fit parameters of the Gaussian modelfit components. The large time-span of the GMVA observations, the fast evolution of the jet, and the limited number of observations make it difficult to robustly cross-identify the Gaussian modelfit components fitted to the three observations. The cross identification of the components is mainly done using morphological similarities and through comparison with near-intime VLBI images at $43 \mathrm{GHz}$ which exhibit similar jet features. The resulting time evolution is shown in Fig. 3. 


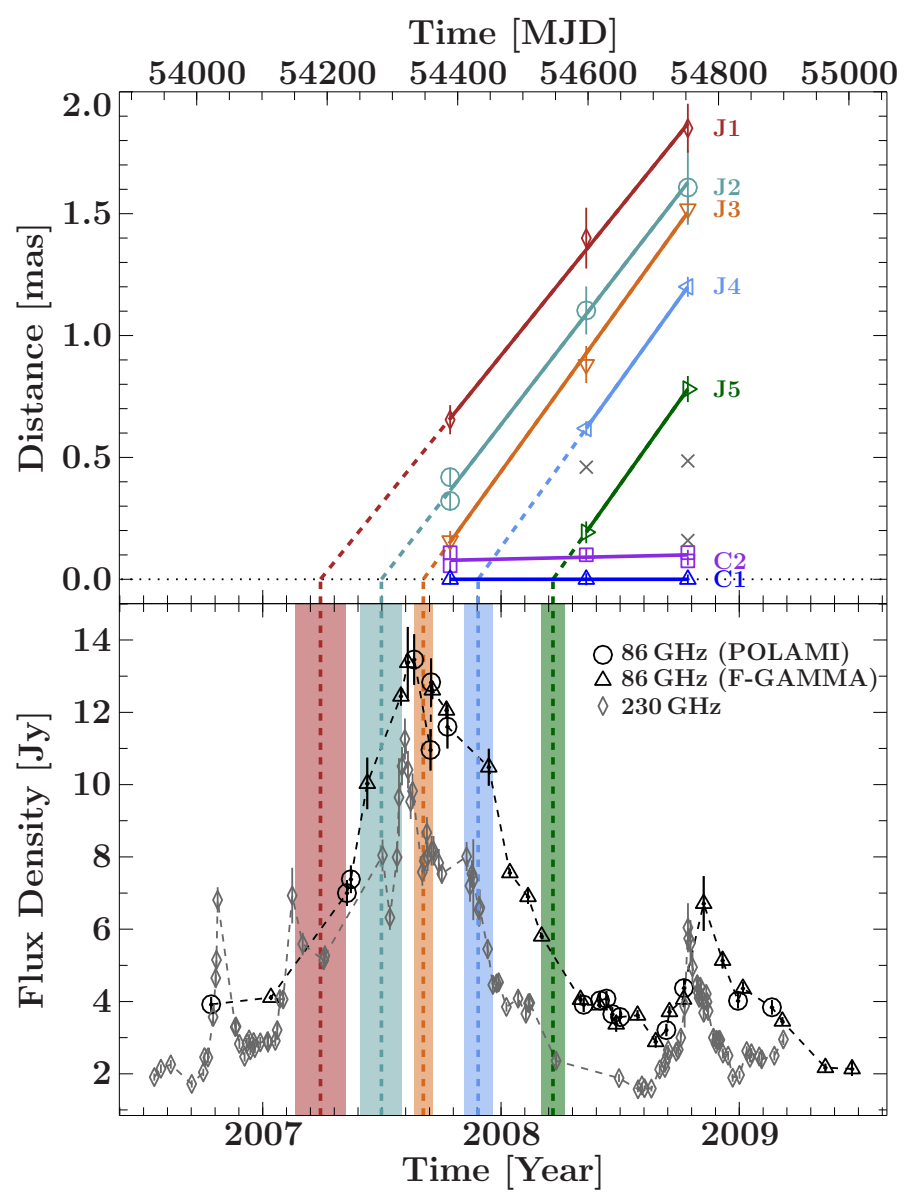

Fig. 1. Upper panel: distance of jet features to the central region over time. Highlighted in color are the associated components and in gray unassociated modelfit components. The solid lines were calculated from the vector fit of the apparent transverse velocity. The colored dashed lines represent extrapolations to the estimated ejection times in the distance-time-domain. Bottom panel: radio light curve at $86 \mathrm{GHz}$ (F-GAMMA, POLAMI) and $230 \mathrm{GHz}$ (Chatterjee et al. 2011) between 01 July 2006 and 01 July 2009. The ejection times of the components are highlighted as vertical, colored, dashed lines in both panels. The wide colored bands indicate the uncertainty in the ejection time of the components.

In all three $86 \mathrm{GHz}$ observations, we find that the most western component which we label as " $\mathrm{C} 1$ " does not correspond to the brightest feature of the jet. We consider it to be the stationary VLBI core and not a sign of a possible counter-jet (see discussion in Sect. 4.3). Therefore, it is used to align the modelfit components at $86 \mathrm{GHz}$ in the subsequent analysis. We label the brightest feature of the jet as component "C2". In the first and last GMVA observations, it is modeled with two, nearby Gaussian model components which are both necessary to represent the associated visibility data, and therefore we treat them as a single entity. For the remaining components of the jet, we assign a "J" plus an integer that increases with the (back-extrapolated) ejection time (see Fig. 1, top panel). In the first epoch, J2 consists of two Gaussian model components which we consider to trace the same emission region due to their proximity to each other.

Figure 4 shows the resulting flux density evolution of $\mathrm{C} 1, \mathrm{C} 2$, and the jet components. There are significant changes in the fluxdensity distribution over time. In the first observation, $\sim 48 \%$ of the total flux density is associated with $\mathrm{C} 1$ and $\mathrm{C} 2$. This fraction decreases slightly in the second observation to $\sim 37 \%$ before

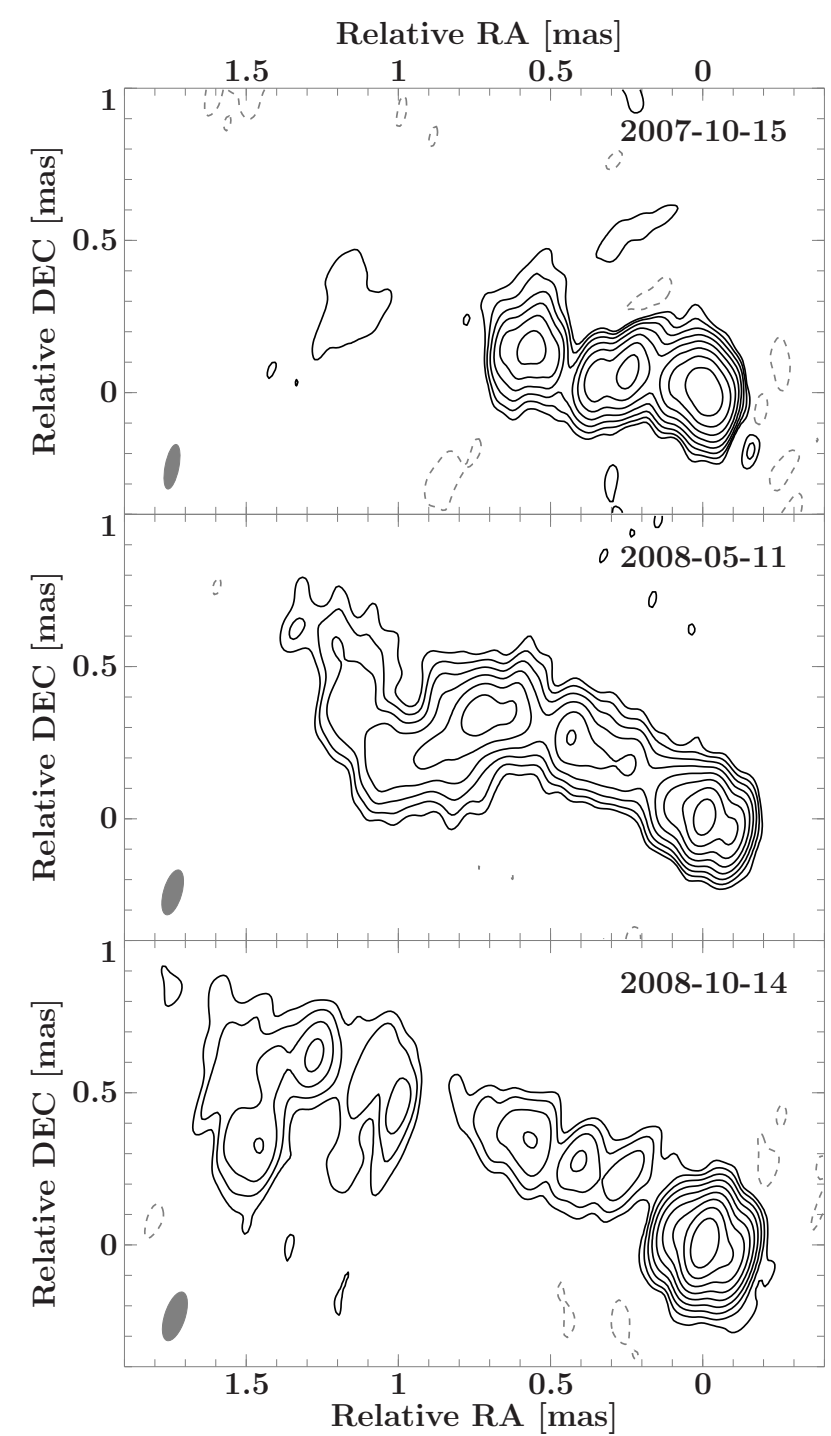

Fig. 2. Images from the three GMVA observations of 3C 111 (image parameters given in Table 1). Contour lines start at three times the individual $1 \sigma$ noise level and increase logarithmically by factors of two. Negative fluctuations at the $-3 \sigma$ level are indicated by dashed, gray contour lines. The gray shaded ellipse in the lower left corner represents the synthesized beam.

increasing to $\sim 74 \%$ in the third epoch. This behavior is consistent with the evolution of the single-dish light curve (Fig. 1), which indicates that the third GMVA observation coincides with the onset of the secondary smaller flare in late 2008. This flare seems to be localized to $\mathrm{C} 1$ and $\mathrm{C} 2$. The brightness temperatures of $\mathrm{C} 1$ and $\mathrm{C} 2$ are of the order of $10^{11} \mathrm{~K}$ in all three epochs (see Table A.2).

We fit the two-dimensional position of the components over time with linear regression in order to determine the angular velocities $v_{x}$ and $v_{y}$. Based on this, we calculated the apparent angular velocities $v_{\text {app }}$. The ejection time was determined from the linear regression in the distance-time domain. The results are listed in Table 2. Care has to be taken in the interpretation of these estimates, considering the limited number of observations at $86 \mathrm{GHz}$.

The results suggest that $\mathrm{C} 2$ is a stationary component consistent with the interpretation of Jorstad et al. (2017) which has strong implications for the jet properties at $15 \mathrm{GHz}$ 


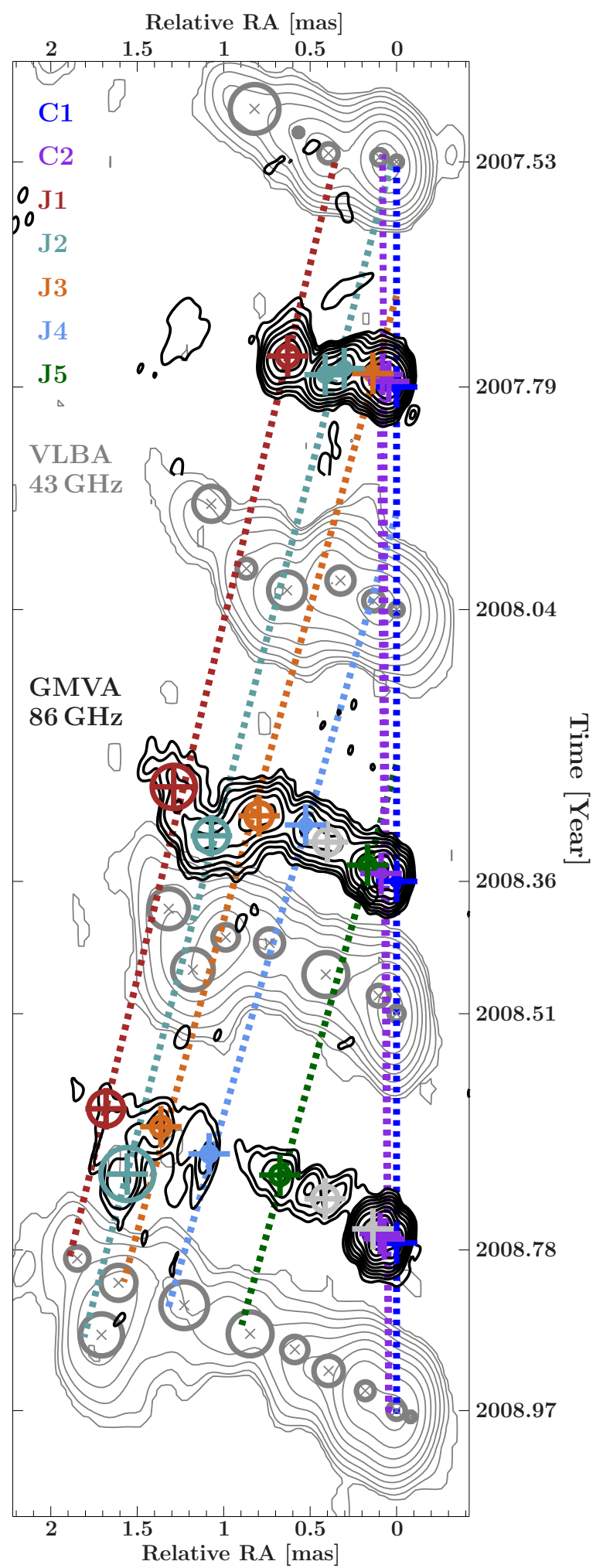

Fig. 3. Time-evolution plot of the source morphology at mm wavelengths. The black solid contour lines are based on the CLEAN-images at $86 \mathrm{GHz}$ (GMVA) after restoring with a common beam $(0.167$ mas $\times$ $\left.0.067 \mathrm{mas},-18.2^{\circ}\right)$ and contour lines starting at three times the noise level. Gaussian model components are superimposed on top of the CLEAN-images. The images and components are aligned to the position of $\mathrm{C} 1$. The gray dashed contour lines show $43 \mathrm{GHz}$ images from the Boston-University blazar group monitoring programme aligned to the position of the core based on model fitting. The colored, dashed lines were calculated from the kinematic fit to the GMVA components and extrapolated to the first and last $43 \mathrm{GHz}$ observation shown here.

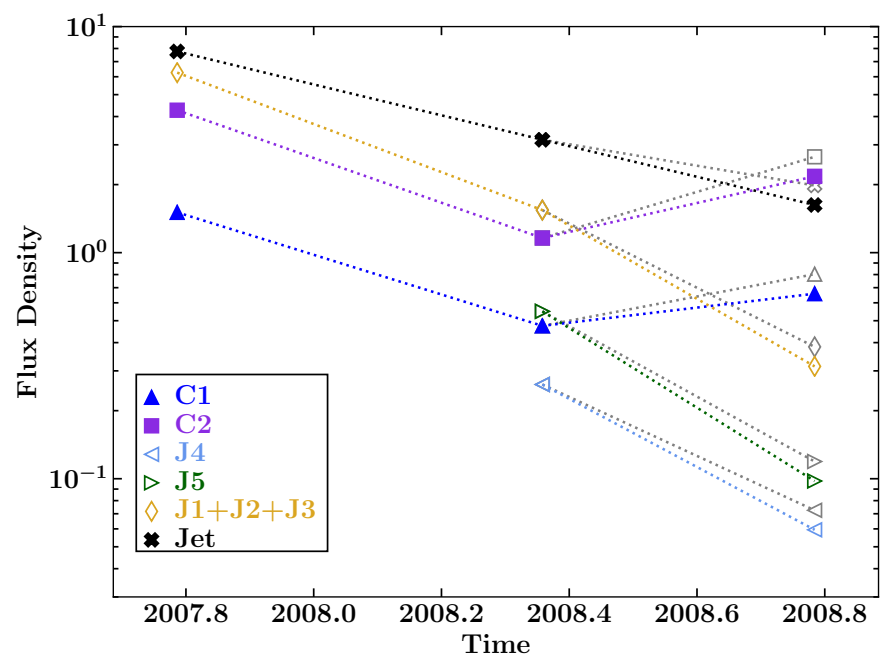

Fig. 4. Flux-density light curves of 3C 111 based on the GMVA data separated into $\mathrm{C} 1, \mathrm{C} 2, \mathrm{~J} 4, \mathrm{~J} 5$, and the sum of the components associated with the bend $(\mathrm{J} 1, \mathrm{~J} 2, \mathrm{~J} 3)$ and the sum of all components representing the jet, that is, all components except $\mathrm{C} 1$ and $\mathrm{C} 2$. For the third VLBI observation, the flux density of the components, if these were scaled using the interpolated scaling factor (see Sect. 2.1) as a systematic uncertainty, is indicated in gray.

Table 2. Kinematic properties of the jet.

\begin{tabular}{lccc}
\hline \hline ID & $\begin{array}{c}v_{\text {app,est }} \\
{\left[\mathrm{mas} \mathrm{yr}^{-1}\right]}\end{array}$ & $\begin{array}{c}\beta_{\text {app,est }} \\
{[\mathrm{c}]}\end{array}$ & $\begin{array}{c}t_{0, \text { est }} \\
{[\mathrm{yr}]}\end{array}$ \\
\hline $\mathrm{C} 2$ & $-0.03 \pm 0.02$ & $-0.09 \pm 0.06$ & \\
$\mathrm{~J} 1$ & $1.2 \pm 0.1$ & $4.0 \pm 0.3$ & $2007.3 \pm 0.1$ \\
$\mathrm{~J} 2$ & $1.3 \pm 0.1$ & $4.2 \pm 0.4$ & $2007.5 \pm 0.1$ \\
$\mathrm{~J} 3$ & $1.36 \pm 0.06$ & $4.5 \pm 0.2$ & $2007.67 \pm 0.04$ \\
$\mathrm{~J} 4$ & $1.4 \pm 0.1$ & $4.5 \pm 0.4$ & $2007.90 \pm 0.06$ \\
$\mathrm{~J} 5$ & $1.4 \pm 0.1$ & $4.5 \pm 0.5$ & $2008.22 \pm 0.05$ \\
\hline
\end{tabular}

(Beuchert et al. 2018). We are able to trace the bend in the jet with two distinct components, $\mathrm{J} 2$ and $\mathrm{J} 3$, which move with an apparent velocity of $\sim 4.2 c$ and $\sim 4.5 c$, respectively. Similar values are also estimated for the other components. We estimate the critical angle $\theta_{\mathrm{LOS} \text {,crit }}$ of the jet to the line of sight (e.g., Cohen et al. 2007), which corresponds to the maximum of $\beta_{\text {app }}$ with $\cos \theta_{\mathrm{LOS}, \text { crit }}=\beta_{\text {app }} / \sqrt{1+\beta_{\text {app }}^{2}}$, to be $\theta_{\mathrm{LOS}, \text { crit }} \sim 13^{\circ}$, consistent with previous estimates at lower frequencies. This leads to a critical Doppler factor $\delta_{\text {crit }}=\gamma_{\min } \sim 4.5$.

The position angle (PA) of $\mathrm{J} 2$ and $\mathrm{J} 3$ differs by $\sim 10^{\circ}$ and $\mathrm{J} 1$ shows a change in PA of $\sim 10^{\circ}$ over time (see Table A.2). The median PA for all components excluding $\mathrm{C} 1$ and $\mathrm{C} 2$ is about $65^{\circ}$ which is consistent with observations at $15 \mathrm{GHz}$ and $43 \mathrm{GHz}$ (Beuchert et al. 2018; Jorstad et al. 2017).

\section{Discussion}

\subsection{Jet evolution and parameters}

The radio light curve at $230 \mathrm{GHz}$ (Fig. 1) reveals that the large outburst began in late 2006 with a small precursor flare occurring shortly before the main flux rise. The top panel of Fig. 1 shows the time evolution of the component distances with time and the back-extrapolated times when they emerged from the 
core. These ejection times coincide well with the characteristic evolution of the light curve at both frequencies. J1, J2, and J3 are the innermost jet components seen in our 2007 GMVA image (and also, at larger distances from the core in later images). Their ejection seems related to the rise and peak of the light curve. The $\mathrm{J} 1$ ejection time falls close to a minor flare seen in the $230 \mathrm{GHz}$ light curve $(65 \mathrm{~d} \pm 37 \mathrm{~d})^{5}$, while $\mathrm{J} 2$ and $\mathrm{J} 3$ are ejected close to the absolute maximum of the outburst $(-49 \mathrm{~d} \pm 37 \mathrm{~d}$ and $-13 \mathrm{~d} \pm 14 \mathrm{~d}$ for $\mathrm{J} 2$ and $\mathrm{J} 3$, respectively at $86 \mathrm{GHz})^{6}$. Here, the $230 \mathrm{GHz}$ light curve again reveals the presence of short-timescale substructure. The ejections of J4 and J5 coincide with the decaying phase of the light curve in late 2007 and early 2008 . While the $86 \mathrm{GHz}$ light curve does not show any specific event here, due to the limited sampling, the $230 \mathrm{GHz}$ data indicate local maxima which correspond well with the ejection times of these two components, in particular with $\mathrm{J} 4$.

The apparent velocities are consistent with the fastest motions seen in previous observations at lower frequencies (Jorstad et al. 2005, 2017; Kadler et al. 2008; Chatterjee et al. 2011; Lister et al. 2013; Beuchert et al. 2018). These studies at $15 \mathrm{GHz}$ and $43 \mathrm{GHz}$ show a broader distribution of $\beta_{\text {app }}$ between $\sim 2 c$ and $\sim 8 c$ while all our $86 \mathrm{GHz}$ components fall into a narrow range in apparent velocities between $(4.0 \pm 0.3) c$ and $(4.5 \pm 0.5) c$ (see Table 2). This might be related to the short time range of our observations which might miss the full extent of activity in 3C 111. Chatterjee et al. (2011) analyzed the $43 \mathrm{GHz}$ observations shown here plus additional ones obtained over a period of 5.4 years, which include the time frame of the flare in 2007 and our GMVA observations. Our results are consistent with those of Chatterjee et al. (2011), except for the association of their component $\mathrm{K} 5$ and the bend (J2, J3, in our model) which were not separated into individual components by Chatterjee et al. (2011).

The critical angle of $\sim 13^{\circ}$ is slightly lower than the average value determined by Jorstad et al. (2017) of $16.3^{\circ} \pm 2.3^{\circ}$ based on variability, and is consistent with the upper limit from Kadler et al. (2008) of $\leq 21^{\circ}$ based on $15 \mathrm{GHz}$ VLBA images. It was noted by Kadler et al. (2008) that these estimates of the angle are in contradiction with the lower limit of $21^{\circ}$ determined for the kiloparsec-scale jet by Lewis et al. (2005). Intriguingly, Hogan et al. (2011) estimated the angle of the large-scale jet to be $\sim 8.1^{\circ}$ based on analysis of the X-ray jet in combination with the parsec-scale jet properties and assuming no deceleration. This is slightly lower than the previous estimates. However, the minimum Lorentz factor determined by Hogan et al. (2011) considering deceleration and bending fits our results, but is below the average value obtained by Jorstad et al. (2017) of $7.7 \pm 0.7$.

Based on $15 \mathrm{GHz}$ VLBI data, Beuchert et al. (2018) report the appearance of a complex feature labeled " $\mathrm{B}$ " as the result of the 2007 outburst which is first detected about 1 mas from the $15 \mathrm{GHz}$ core in their $15 \mathrm{GHz}$ VLBA analysis. It is shown to evolve into multiple components, B1, B2, B3, and B4, which seem to correspond to $\mathrm{J} 1, \mathrm{~J} 2, \mathrm{~J} 3$, and $\mathrm{J} 4$ at $86 \mathrm{GHz}$ as shown in Fig. 5. The $15 \mathrm{GHz}$ modelfit components are well aligned with the $43 \mathrm{GHz}$ components (see also Table A.3) with small differences being most likely the result of the different resolution. The difference in position between $43 \mathrm{GHz}$ and $86 \mathrm{GHz}$ components (see Table A.3) can be explained best by the movement of the jet

\footnotetext{
5 Based on the estimated ejection date and the flux density measurement at $230 \mathrm{GHz}$ on 2007 Feb. 15.

6 Based on the estimated ejection date and the flux measurement at $86 \mathrm{GHz}$ on 2007 Aug. 02.
}

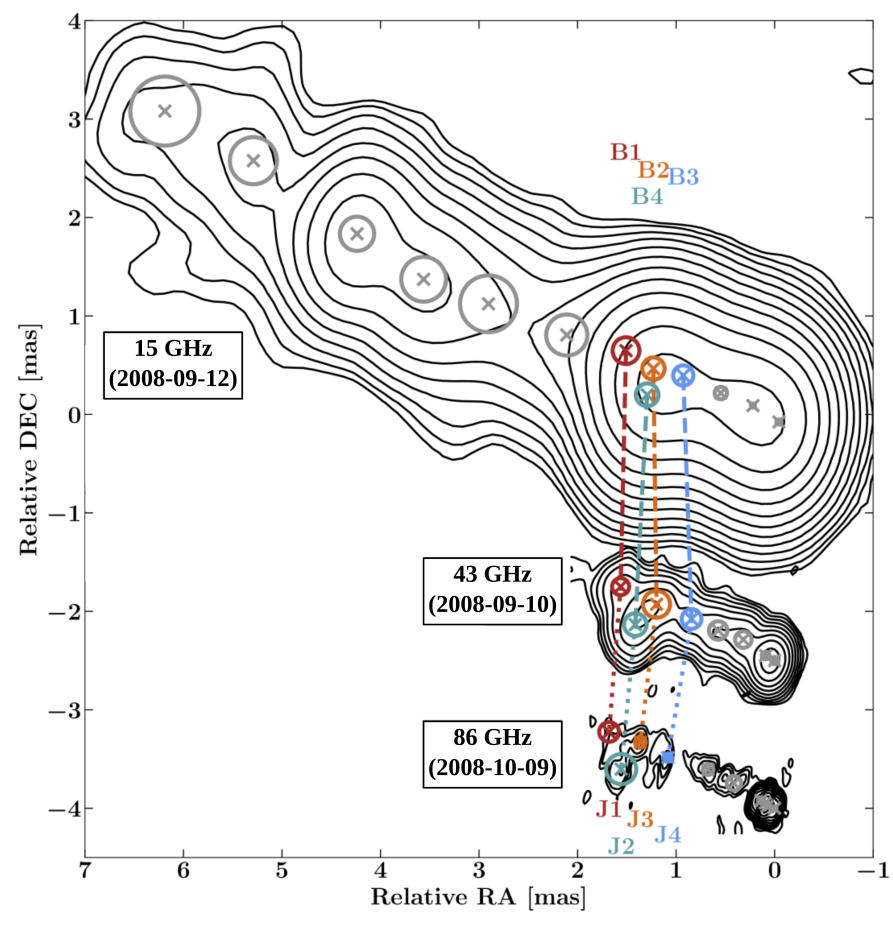

Fig. 5. Multifrequency images of $3 \mathrm{C} 111$ at $15 \mathrm{GHz}$ (VLBA, from the MOJAVE programme), $43 \mathrm{GHz}$ (VLBA, from the Boston-Blazar-Group programme) and $86 \mathrm{GHz}$ (GMVA) to highlight the association of J1, J2, $\mathrm{J} 3$ and $\mathrm{J} 4$ with the $15 \mathrm{GHz}$ counterparts (B1, B2, B3, B4) reported in Beuchert et al. (2018). In this image, we corrected for the core shift between $15 \mathrm{GHz}$ and $43 \mathrm{GHz}$ by applying a shift to the $15 \mathrm{GHz}$ image $(\Delta \mathrm{RA}=-0.22$ mas, $\Delta \mathrm{Dec}=-0.09$, Beuchert et al. 2018). The coordinates of the components are listed in Table A.3.

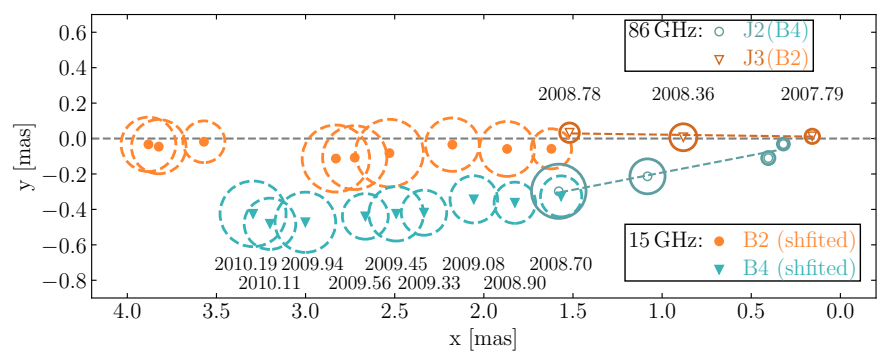

Fig. 6. Modelfit components $\mathrm{J} 2$ and $\mathrm{J} 3$ at $86 \mathrm{GHz}$, and $\mathrm{B} 2$ and $\mathrm{B} 4$ at $15 \mathrm{GHz}$ from Beuchert et al. (2018) after rotation by the median position angle of the jet of $65^{\circ}$. B2 and B4 are corrected for the core shift between $15 \mathrm{GHz}$ and $43 \mathrm{GHz}$.

features as show in Fig. 3. This consistency makes it possible to trace the future evolution of components $\mathrm{J} 2$ and $\mathrm{J} 3$ to larger distances than would have been possible at $86 \mathrm{GHz}$ alone. This is illustrated in Fig. 6 (see also Beuchert et al. 2018), which shows the components $\mathrm{J} 2$ and $\mathrm{J} 3$ in combination with $\mathrm{B} 2$ and $\mathrm{B} 4$ from Beuchert et al. (2018) rotated by the median jet position angle of $65^{\circ}$ and corrected for the core shift between $15 \mathrm{GHz}$ and $43 \mathrm{GHz}$. The core shift between $43 \mathrm{GHz}$ and $86 \mathrm{GHz}$ is very likely much smaller than the one from $15 \mathrm{GHz}$ to $43 \mathrm{GHz}$ as has been shown by other studies of AGNs (e.g., Lobanov 1998; Hada et al. 2011; Fromm et al. 2013b).

Based on Fig. 6 it seems likely that $\mathrm{J} 2 / \mathrm{J} 3$ at $86 \mathrm{GHz}$ and B2/B4 at $15 \mathrm{GHz}$ can be associated with each other. This association has significant implications for the evolution of this jet structure which is discussed in Sect. 4.4. 


\subsection{Comparison with the outburst in 1996}

As mentioned in Sect. 1, a major outburst was recorded in early 1996 similar to the one in 2007 in magnitude (Alef et al. 1998). Alef et al. (1998) and Kharb et al. (2003) presented $43 \mathrm{GHz}$ VLBA images taken in 1996 July and 1996 Sep. The morphology indicates a small bend in the jet similar to the $43 \mathrm{GHz}$ images in 2008.04 shown in Fig. 3. In the $43 \mathrm{GHz}$ images obtained in 1998, Jorstad et al. (2005) detected a large diffuse structure located beyond $\sim 2$ mas from the core. The study concludes that this structure might represent the remnant of the 1996 outburst and measured $\beta_{\text {app }} \approx 6 c$ for its propagation. In addition, Jorstad et al. (2005) reported significant polarization in this feature at $43 \mathrm{GHz}$.

The $43 \mathrm{GHz}$ images presented by Chatterjee et al. (2011) suggest that the feature related to the 2007 outburst has traveled a similar distance in a similar time range becoming increasingly diffuse and extended. At $15 \mathrm{GHz}$, Beuchert et al. (2018) show that feature $B$ likewise becomes increasingly complex with time, eventually dominating the polarization of the entire jet.

These are all indications that the major outbursts in 1996 and 2007 represent similar events that differ from intermediate, minor flares from this radio source. Based on this, it seems probable that a similar event will occur in the future. If the $\gamma$-ray emission in $3 \mathrm{C} 111$ is correlated to the radio activity as has been seen in other sources, then a future large radio outburst would represent a prime opportunity to study the radio- $\gamma$-ray connection. Grandi et al. (2012) associated the $\gamma$-ray emission detected in 3C 111 in 2008 Oct./Nov. with a TS-value of 9.7 with a small flare in late 2008 (see Fig. 1). If this connection holds, then a larger radio outburst may coincide with a larger $\gamma$-ray event.

Interestingly, components $\mathrm{J} 4$ and $\mathrm{J} 5$, which are trailing $\mathrm{J} 1-\mathrm{J} 3$, have a much lower flux density than the sum of bendrelated components $\mathrm{J} 1, \mathrm{~J} 2$, and $\mathrm{J} 3$ (Fig. 4). This behavior is in agreement with the modeling of the 1996 flare in Kadler et al. (2008) and Perucho et al. (2008). In those works, the authors suggested that the major flares are followed by drops in the mass flux being injected into the jet, resulting in a decrease in brightness. Figure 4 also indicates a steeper drop in flux density from the second to third epoch compared to the drop from the first to second epoch. This steeper drop cannot be explained by the flux density normalization of epoch 3 even if the systematic uncertainty is taken into account. However, because of the limited number of observations over the long time frame, we cannot undertake a detailed physical interpretation.

\subsection{The nature of $C 1$}

Traditionally, the brightest feature in the jet of 3C 111 corresponded to the westernmost component. Here, we find an additional component upstream of the brightest emission region. Such a component was reported by Grandi et al. (2012) based on super-resolved $43 \mathrm{GHz}$ VLBA images. The study interpreted the brightest feature as the core of the jet and the component upstream of the core as the counter-jet based on the stationary behavior, but no detailed analysis was performed.

Various arguments seem to disfavor this scenario. In particular, using the ratio $R$ of the flux density of the jet and counter-jet, it is possible to estimate $\theta_{\mathrm{LOS}}$ for a given intrinsic speed $\beta$ and a continuous, intrinsically symmetric jet (e.g., Urry \& Padovani 1995) via

$R=\left(\frac{1+\beta \cos \theta_{\mathrm{LOS}}}{1-\beta \cos \theta_{\mathrm{LOS}}}\right)^{2+\alpha}$,

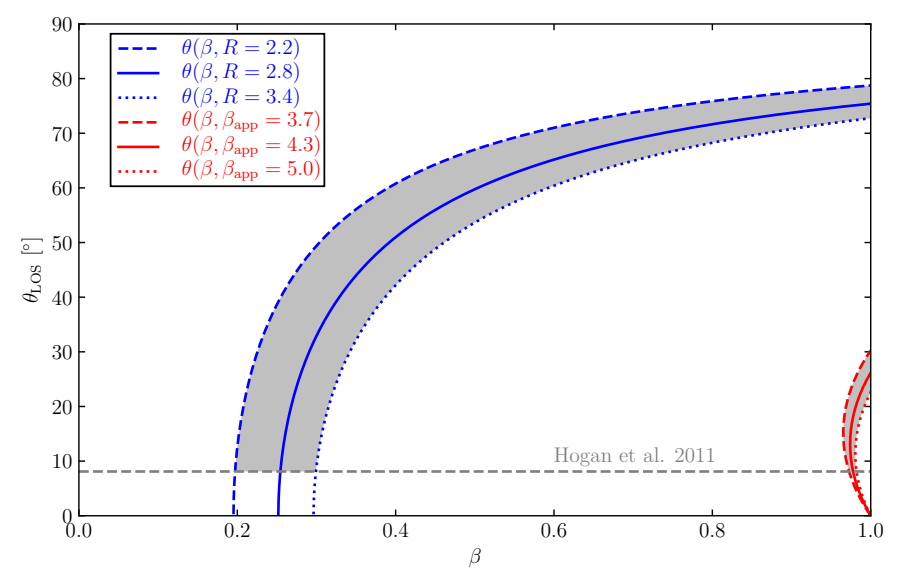

Fig. 7. Angle between the jet and the line of sight $\theta_{\mathrm{LOS}}$ for a given intrinsic speed $\beta$, for the flux density ratio of the $\mathrm{C} 1$ and $\mathrm{C} 2$ components (blue) and the range of measured apparent velocities (red) of $3 \mathrm{C} 111$. The gray shaded areas represent the parameter space for $\theta_{\mathrm{LOS}}$ and $\beta$ for both cases. The gray dashed line is a lower limit of the large-scale $\theta_{\mathrm{LOS}}$ from Hogan et al. (2011).

where $\alpha$ is the spectral index. Assuming $\mathrm{C} 2$ and $\mathrm{C} 1$ are actually the VLBI cores of the jet and counter-jet, the corresponding flux densities in the first epoch yield $R \sim 2.8$. The gray-shaded area marked by the blue lines in Fig. 7 shows the resulting possible parameter space of $\theta_{\text {LOS }}$ and $\beta$ accounting for the uncertainty of the flux-density calibration and the lower limit on $\theta_{\mathrm{LOS}}$ estimated by Hogan et al. (2011). An independent way of constraining both parameters is using $\beta_{\text {app }}$ from the previous section:

$\beta_{\text {app }}=\frac{\beta \sin \theta_{\mathrm{LOS}}}{1-\beta \cos \theta_{\mathrm{LOS}}}$

This yields the gray-shaded area between the red lines in Fig. 7 which shows no intersection with the gray-shaded area representing the allowed parameter space from Eq. (1). Solving this discrepancy would require a large change in $\theta_{\mathrm{LOS}}$ on scales below the resolution capabilities of the GMVA, which seems unlikely given that the parsec- and kiloparsec-scale jet are well aligned and remarkably straight. Alternatively, the scenario suggested by Grandi et al. (2012) would require unusually large intrinsic acceleration over the observed short distance. The reported angles and Doppler factors for 3C 111 show that the radio emission is significantly boosted with $S_{\text {int }}=\delta^{3+\alpha} S_{\text {obs }}$, and any counter-jet emission is expected to be de-boosted by $1 / \delta^{3+\alpha}$. For $\alpha=0$, this would require $\delta \sim 1.2$ to account for the given $R$ which is significantly below estimates from the kinematics of the jet. While we cannot entirely exclude the possibility of high intrinsic acceleration, we do not consider it to be the case here based on the arguments above. Therefore, we favor the interpretation that $\mathrm{C} 2$ represents a stationary component close to the core (C1) based on these arguments which is consistent with conclusions of Jorstad et al. (2017) This means that all modelfit components belong to the jet and no counter is visible which would correspond to much higher $R$ value and a lower $\theta_{\text {LOS }}$.

In the canonical jet model (Blandford \& Königl 1979; Königl 1981), the core corresponds to the transition region of the jet from synchrotron self-absorbed to optically thin emission where the opacity reaches $\tau=1$. Another possibility is that it represents a recollimation shock (e.g., Daly \& Marscher 1988; Gómez et al. 1995, 1997; Fromm et al. 2011, 2013b; Marscher 2014). In the case of components $\mathrm{C} 1$ and $\mathrm{C} 2$, a core-shift analysis is necessary in order to clarify whether $\mathrm{C} 1$ is a recollimation 
shock or not. In case of a recollimation shock, the core shift would not decrease with increasing frequency (Fromm et al. 2018). Future quasi-simultaneous multi-frequency VLBI observations up to $1 \mathrm{~mm}$ wavelength might be able to test this.

It is important to point out that our kinematic results only marginally depend on whether $\mathrm{C} 1$ or $\mathrm{C} 2$ is used for alignment as they are both kinematically stationary. Given the small distance between both components, we can use $\mathrm{C} 1$ as the reference point.

\subsection{The transversal structure of the jet}

Figure 6 shows the distinct trajectory of component J2/B4 with respect to component J3/B2 and the link to other $15 \mathrm{GHz}$ components from Beuchert et al. (2018). There is a clear connection between the components: (1) the $15 \mathrm{GHz}$ jet represents a smooth continuation of the $86 \mathrm{GHz}$ jet on larger scales, and (2) the long-term kinematics of the components are different when the $15 \mathrm{GHz}$ evolution is considered, depending on the direction of propagation. Although components $\mathrm{J} 2$ and $\mathrm{J} 3$ show similar velocities at the observed $86 \mathrm{GHz}$ epochs, J2 decelerates with respect to $\mathrm{J} 3$ farther downstream. In relation with the originating flare, this process shows that the flare has substructure in space and time.

The growth of the observed jet bend might be interpreted as the development of a kink instability along the jet because of the flare (Tchekhovskoy \& Bromberg 2016). The structure is observed to grow out to a distance of $r \simeq 3.5$ mas at $15 \mathrm{GHz}$, with a maximum amplitude of 0.5 mas. However, the $15 \mathrm{GHz}$ kinematics show that this large amplitude does not trigger jet deceleration which would be expected following the disruption in the jet stream. In addition, the overall jet remains remarkably collimated on larger scales. Therefore, unless the instability and disruption develop at the boundary between the jet spine and the surrounding sheath, we regard the kink-instability option as improbable.

The $230 \mathrm{GHz}$ light-curve shown in Fig. 1 shows that the global evolution is modulated by short-scale spikes and drops. The five local maxima observed along the outburst can be related, within errors, to the ejection of the different modeled components. Components J1/B1 and J3/B2 propagate along the spine, whereas component J2/B4 moves along a different PA (see Fig. 6). Components J4 and J5 also propagate along the spine (at least out to a distance of 1.5 mas covered by our GMVA data).

Therefore, the ejection of bright components seems to happen in a discrete way when the launching region becomes active. It is already known that the modeled radio components associated to bright regions propagating down the jet are ejected with different PAs (Beuchert et al. 2018; Lister et al. 2013) and that only stacked images combining a number of VLBI observations can reveal the whole time-averaged volume covered by the radio jet. Our observations probably reveal such behavior within a single flare.

The cross-identification of components from the $86 \mathrm{GHz}$ and the $15 \mathrm{GHz}$ images (see Fig. 6) allows us to follow the trajectory and kinematics of these components farther downstream of the $86 \mathrm{GHz}$ jet. We observe that component J2/B4 not only follows a trajectory along the bottom of the jet, but is also decelerated with respect to the components moving along the spine of the jet. It therefore seems that $\mathrm{J} 2 / \mathrm{B} 4$ is decelerating due to direct interaction with the ambient medium.

We can use the distance of $\mathrm{J} 2$ and $\mathrm{J} 3 r_{\mathrm{J} 2 \mathrm{~J} 3}$ in the last GMVA epoch as shown in Fig. 6, including the size of the components, to estimate the deprojected opening angle $\psi$ following $\tan \psi=r_{\mathrm{J} 2, \mathrm{~J} 3} / d_{\text {proj,J3 }} \times \sin \theta_{\mathrm{LOS}}$, where $d_{\text {proj,J3 }}$ corresponds to the projected distance of J3. With $8^{\circ} \lesssim \theta_{\text {LOS }} \lesssim 30^{\circ}$, this yields $3.8^{\circ} \lesssim \psi \lesssim 13^{\circ}$, but the actual value may be larger due to possible further extended emission below the sensitivity limit. The range of $\psi$ is consistent with average values determined at $43 \mathrm{GHz}$ by Jorstad et al. (2017) using two different methods, that is, $4.6^{\circ} \pm 1.2^{\circ}$ and $8.8^{\circ} \pm 6.2^{\circ}$.

In summary, the role that such peripheral components such as J2/B4 play is two-fold. On the one hand, they carve the channel boundaries that allow the faster propagation along the jet spine. Additionally, they may be the origin of the jet transversal structure, that is, of a spine-sheath structure within the radio jet. We want to stress that such layers increase the jet stability (e.g., Perucho et al. 2005; Perucho \& Lobanov 2007; Martí et al. 2016; Perucho 2019).

\section{Summary and conclusions}

In this paper, we present three GMVA observations at $86 \mathrm{GHz}$ of the $\gamma$-ray-loud radio galaxy $3 \mathrm{C} 111$ that were obtained over a period of one year shortly after a major outburst above $10 \mathrm{Jy}$ in 2007. The mm-wavelength light curves show that the whole flare covered a time range of more than a year starting in late 2006 and decaying well into 2008 with minor flares before and after the peak.

The high dynamic range of the GMVA images reveals a complex morphology with a distinct feature in the form of a bend in the otherwise well-collimated jet. The images and Gaussian components fitted to the data reveal an emission region upstream of the brightest feature of the jet. We consider the upstream emission as the core of the jet and not part of the counter jet. Both features are stationary and have high brightness temperatures of up to $3 \times 10^{11} \mathrm{~K}$. One of them may be interpreted as part of a recollimation shock, but further multi-frequency VLBI observations at $86 \mathrm{GHz}$ and above are necessary.

We combine the GMVA data with $43 \mathrm{GHz}$ VLBA images to constrain the time evolution of the jet at $86 \mathrm{GHz}$, which suggests that all features of the jet move with apparent velocities between $\sim 4.0 c$ and $\sim 4.5 c$. The maximum speed corresponds to a critical angle of the jet to the line of sight of $\sim 13^{\circ}$ and a critical Doppler factor of $\sim 4.5$, consistent with previous studies. The high angular resolution of the GMVA observations enables us to separate the observed bend into two distinct features, $\mathrm{J} 2$ and $\mathrm{J} 3$, which have estimated ejection times that correspond well to the maximum of the flare. Our results show that a kinematic study is possible even with the two observation windows per year of the GMVA for such a fast-evolving jet as 3C 111 if the GMVA observations are supported with interleaved $43 \mathrm{GHz}$ monitoring.

We can associate the features of the bend down to $15 \mathrm{GHz}$ allowing us to track them over a larger distance. The VLBI data in combination with the radio light curves suggest that these latter features originated from distinct ejections into the jet stream throughout the increased activity cycle of the AGN. The evolution of the bend indicates that we trace the transversal structure of the jet in terms of a spine and sheath as a result of the outburst.

To date, 3C 111 has exhibited only one other major radio flare (in 1996) that matches the scale of this radio outburst in 2007. Only a much smaller radio outburst occurred in-between these two events. It is therefore plausible that a similar major event will take place in the future. Since a connection between a minor radio flare and the $\gamma$-ray activity has been proposed in the literature (Grandi et al. 2012), it is interesting to consider that a future outburst similar to the one in 2007 could lead to increased high-energy activity. Hence, 3C 111 has the potential to be a key target source for studies that probe the origin and development of 
the smallest substructures in AGN jets that lead to the enigmatic short-term variability observed in some blazar jets. At an intermediate angle between classical blazars and radio galaxies and with its bright mm-band, compact jet emission at small redshift, 3C 111 offers the possibility for high-resolution multiwavelength studies of AGN-jet variability during high-energy flares.

Acknowledgements. We would like to thank the anonymous referee for helpful comments that improved the manuscript. We would like to thank the internal MPIfR referee N. MacDonald for insightful comments that helped improve the manuscript. RS gratefully acknowledge support from the European Research Council under the European Union's Seventh Framework Programme (FP/20072013)/ERC Advanced Grant RADIOLIFE-320745 and support by Deutsche Forschungsgemeinschaft grant WI 1860/10-1. MP acknowledges financial support from the Spanish Ministry of Science through Grants PID2019-105510GBC31, PID2019-107427GB-C33 and AYA2016-77237-C3-3-P, and from the Generalitat Valenciana through grant PROMETEU/2019/071. I.A. acknowledges support by a Ramón y Cajal grant (RYC-2013-14511) of the "Ministerio de Ciencia e Innovación (MICINN)" of Spain. He also acknowledges financial support from MCINN through the "Center of Excellence Severo Ochoa" award for the Instituto de Astrofísica de Andalucía-CSIC (SEV-2017-0709). Acquisition and reduction of the POLAMI data was supported in part by MICINN through grant AYA2016-80889-P. IRAM is supported by INSU/CNRS (France), MPG (Germany) and IGN (Spain). This research has made use of data obtained with the Global Millimeter VLBI Array (GMVA), which consists of telescopes operated by the MPIfR, IRAM, Onsala, Metsahovi, Yebes, the Korean VLBI Network, the Green Bank Observatory and the Very Long Baseline Array (VLBA). The VLBA is a facility of the National Science Foundation operated under cooperative agreement by Associated Universities, Inc. The data were correlated at the correlator of the MPIfR in Bonn, Germany. This study makes use of $43 \mathrm{GHz}$ VLBA data from the Boston University gamma-ray blazar monitoring program (http: //www.bu.edu/blazars/VLBAproject.html), funded by NASA through the Fermi Guest Investigator Program. This research has made use of data from the MOJAVE database that is maintained by the MOJAVE team (Lister et al. 2009). The Very Long Baseline Array (VLBA) is an instrument of the National Radio Astronomy Observatory (NRAO). NRAO is a facility of the National Science Foundation, operated by Associated Universities Inc. This research made use of the Interactive Spectral Interpretation System (ISIS) (Houck \& Denicola 2000) and a collection of ISIS scripts provided by the Dr. Karl Remeis observatory, Bamberg, Germany at http://www.sternwarte.uni-erlangen.de/isis/. This research made use of the NASA/IPAC Extragalactic Database (NED) which is operated by the Jet Propulsion Laboratory, California Institute of Technology, under contract with the National Aeronautics and Space Administration and of the VizieR catalogue access tool, CDS, Strasbourg, France.

\section{References}

Agudo, I., Jorstad, S. G., Marscher, A. P., et al. 2011, ApJ, 726, L13 Agudo, I., Thum, C., Gómez, J. L., \& Wiesemeyer, H. 2014, A\&A, 566, A59 Agudo, I., Thum, C., Molina, S. N., et al. 2018a, MNRAS, 474, 1427 Agudo, I., Thum, C., Ramakrishnan, V., et al. 2018b, MNRAS, 473, 1850 Aharonian, F., Akhperjanian, A. G., Bazer-Bachi, A. R., et al. 2007, ApJ, 664, L71

Albert, J., Aliu, E., Anderhub, H., et al. 2007, ApJ, 669, 1143

Alef, W., Preuss, E., Kellermann, K. I., \& Gabuzda, D. 1998, in IAU Colloq 164: Radio Emission from Galactic and Extragalactic Compact Sources, eds. J. A. Zensus, G. B. Taylor, \& J. M. Wrobel, ASP Conf. Ser., 144, 129

Aleksić, J., Antonelli, L. A., Antoranz, P., et al. 2014, A\&A, 563, A91

Angelakis, E., Fuhrmann, L., Nestoras, I., et al. 2010, ArXiv e-prints [arXiv:1006.5610]

Angelakis, E., Fuhrmann, L., Nestoras, I., et al. 2012, J. Phys. Conf. Ser., 372, 012007

Angelakis, E., Fuhrmann, L., Myserlis, I., et al. 2019, A\&A, 626, A60 Beuchert, T., Rodríguez-Ardila, A., Moss, V. A., et al. 2018, A\&A, 612, L4 Blandford, R. D., \& Königl, A. 1979, ApJ, 232, 34

Boccardi, B., Krichbaum, T. P., Ros, E., \& Zensus, J. A. 2017, A\&ARv, 25, 4 Casadio, C., Gómez, J. L., Grandi, P., et al. 2015, ApJ, 808, 162

Chatterjee, R., Marscher, A. P., Jorstad, S. G., et al. 2011, ApJ, 734, 43

Cohen, M. H., Lister, M. L., Homan, D. C., et al. 2007, ApJ, 658, 232

Daly, R. A., \& Marscher, A. P. 1988, ApJ, 334, 539
Fanaroff, B. L., \& Riley, J. M. 1974, MNRAS, 167, 31P

Fomalont, E. B. 1999, in Synthesis Imaging in Radio Astronomy II, eds. G. B. Taylor, C. L. Carilli, \& R. A. Perley, ASP Conf. Ser., 180, 301

Freedman, W. L., Madore, B. F., Gibson, B. K., et al. 2001, ApJ, 553, 47

Fromm, C. M., Perucho, M., Ros, E., et al. 2011, A\&A, 531, A95

Fromm, C. M., Ros, E., Perucho, M., et al. 2013a, A\&A, 551, A32

Fromm, C. M., Ros, E., Perucho, M., et al. 2013b, A\&A, 557, A105

Fromm, C. M., Perucho, M., Porth, O., et al. 2018, A\&A, 609, A80

Fuhrmann, L., Larsson, S., Chiang, J., et al. 2014, MNRAS, 441, 1899

Fuhrmann, L., Angelakis, E., Zensus, J. A., et al. 2016, A\&A, 596, A45

Gómez, J. L., Marti, J. M. A., Marscher, A. P., Ibanez, J. M. A., \& Marcaide, J. M. 1995, ApJ, 449, L19

Gómez, J. L., Martí, J. M., Marscher, A. P., Ibáñez, J. M., \& Alberdi, A. 1997, ApJ, 482, L33

Götz, M. M. A., Alef, W., Preuss, E., \& Kellermann, K. I. 1987, A\&A, 176, 171 Grandi, P., Torresi, E., \& Stanghellini, C. 2012, ApJ, 751, L3

Greisen, E. W. 2003, Information Handling in Astronomy - Historical Vistas (Dordrecht: Kluwer Academic Publishers), 285, 109

Hada, K., Doi, A., Kino, M., et al. 2011, Nature, 477, 185

Hartman, R. C., Kadler, M., \& Tueller, J. 2008, ApJ, 688, 852

Hogan, B. S., Lister, M. L., Kharb, P., Marshall, H. L., \& Cooper, N. J. 2011, ApJ, 730, 92

Högbom, J. A. 1974, A\&AS, 15, 417

Houck, J. C., \& Denicola, L. A. 2000, in Astronomical Data Analysis Software and Systems IX, eds. N. Manset, C. Veillet, \& D. Crabtree, ASP Conf. Ser., 216,591

Hovatta, T., Nieppola, E., Tornikoski, M., et al. 2008, A\&A, 485, 51

Hovatta, T., Valtaoja, E., Tornikoski, M., \& Lähteenmäki, A. 2009, A\&A, 494, 527

Jorstad, S. G., Marscher, A. P., Mattox, J. R., et al. 2001, ApJ, 556, 738

Jorstad, S. G., Marscher, A. P., Lister, M. L., et al. 2005, AJ, 130, 1418

Jorstad, S. G., Marscher, A. P., Morozova, D. A., et al. 2017, ApJ, 846, 98

Kadler, M., Ros, E., Perucho, M., et al. 2008, ApJ, 680, 867

Karamanavis, V., Fuhrmann, L., Krichbaum, T. P., et al. 2016, A\&A, 586, A60

Kharb, P., Gabuzda, D., Alef, W., Preuss, E., \& Shastri, P. 2003, New Astron. Rev., 47, 621

Königl, A. 1981, ApJ, 243, 700

Lähteenmäki, A., \& Valtaoja, E. 1999, ApJ, 521, 493

Lähteenmäki, A., \& Valtaoja, E. 2003, ApJ, 590, 95

León-Tavares, J., Valtaoja, E., Tornikoski, M., Lähteenmäki, A., \& Nieppola, E. 2011, A\&A, 532, A146

Lewis, K. T., Eracleous, M., Gliozzi, M., Sambruna, R. M., \& Mushotzky, R. F. 2005, ApJ, 622, 816

Linfield, R., \& Perley, R. 1984, ApJ, 279, 60

Lister, M. L., Homan, D. C., Kadler, M., et al. 2009, ApJ, 696, L22

Lister, M. L., Aller, M. F., Aller, H. D., et al. 2013, AJ, 146, 120

Lobanov, A. P. 1998, A\&A, 330, 79

Marscher, A. P. 2014, ApJ, 780, 87

Marscher, A. P., \& Gear, W. K. 1985, ApJ, 298, 114

Marscher, A. P., Jorstad, S. G., Larionov, V. M., et al. 2010, ApJ, 710, L126

Marscher, A., Jorstad, S. G., Larionov, V. M., Aller, M. F., \& Lähteenmäki, A. 2011, J. Astrophys. Astron., 32, 233

Martí, J. M., Perucho, M., \& Gómez, J. L. 2016, ApJ, 831, 163

Perucho, M. 2019, Galaxies, 7, 70

Perucho, M., \& Lobanov, A. P. 2007, A\&A, 469, L23

Perucho, M., Martí, J. M., \& Hanasz, M. 2005, A\&A, 443, 863

Perucho, M., Agudo, I., Gómez, J. L., et al. 2008, A\&A, 489, L29

Richards, J. L., Hovatta, T., Max-Moerbeck, W., et al. 2014, MNRAS, 438, 3058

Savolainen, T., Wiik, K., Valtaoja, E., Jorstad, S. G., \& Marscher, A. P. 2002, A\&A, 394, 851

Schinzel, F. K., Lobanov, A. P., Taylor, G. B., et al. 2012, A\&A, 537, A70

Schulz, R., Kadler, M., Ros, E., et al. 2013, ArXiv e-prints [arXiv:1301.6582]

Schwab, F. R., \& Cotton, W. D. 1983, AJ, 88, 688

Shepherd, M. C. 1997, in Astronomical Data Analysis Software and Systems VI, eds. G. Hunt, \& H. Payne, ASP Conf. Ser., 125, 77

Tchekhovskoy, A., \& Bromberg, O. 2016, MNRAS, 461, L46

Thum, C., Agudo, I., Molina, S. N., et al. 2018, MNRAS, 473, 2506

Trippe, S., Krips, M., Piétu, V., et al. 2011, A\&A, 533, A97

Urry, C. M., \& Padovani, P. 1995, PASP, 107, 803

Valtaoja, E., Lähteenmäki, A., Teräsranta, H., \& Lainela, M. 1999, ApJS, 120, 95

Véron-Cetty, M.-P., \& Véron, P. 2010, A\&A, 518, A10 


\section{Appendix A: Additional tables}

Table A.1. GMVA stations that participated in our observations in 2007 and 2008.

\begin{tabular}{lccccc}
\hline \hline Station & $\begin{array}{c}\text { Diameter } \\
{[\mathrm{m}]}\end{array}$ & $\begin{array}{c}\text { SEFD } \\
{[\mathrm{Jy}]}\end{array}$ & $\begin{array}{c}\text { Epoch 1 } \\
\text { 2007-10-13 }\end{array}$ & $\begin{array}{c}\text { Epoch 2 } \\
\text { 2008-05-11 }\end{array}$ & $\begin{array}{c}\text { Epoch 3 } \\
\text { 2008-10-14 }\end{array}$ \\
\hline Metsähovi & 14 & 17647 & $\checkmark$ & $\checkmark^{(d)}$ & X \\
Onsala & 20 & 6122 & $\checkmark$ & $\checkmark$ & $\checkmark$ \\
Effelsberg & 100 & 929 & $\checkmark$ & $\checkmark$ & $\checkmark$ \\
Plateau de Bure & $35^{(a)}$ & 409 & $\checkmark$ & $\checkmark^{(d)}$ & X \\
Pico Veleta $^{(b)}$ & 30 & 643 & $\checkmark^{(c)}$ & $\checkmark$ & $\checkmark^{(e)}$ \\
$8 \times$ VLBA $^{(b)}$ & 25 & 2941 & $\checkmark$ & $\checkmark$ & $\checkmark^{(f)}$ \\
\hline
\end{tabular}

Notes. ${ }^{(a)}$ Equivalent diameter of a phased interferometric array with $6 \times 15 \mathrm{~m}$ telescopes. ${ }^{(b)}$ The eight VLBA stations are North Liberty, Fort Davis, Los Alamos, Pie Town, Kit Peak, Owens Valley, Brewster, Mauna Kea. ${ }^{(c)}$ No fringes after correlation. ${ }^{(d)}$ Flagged during a-priori calibration. ${ }^{(e)}$ Data were lost due to bad weather. ${ }^{(f)}$ Data of North Liberty and Fort Davis were flagged during hybrid imaging.

Table A.2. Parameters of the GMVA jet components prior to positional alignment.

\begin{tabular}{|c|c|c|c|c|c|}
\hline $\operatorname{ID}^{(a)}$ & $\begin{array}{c}S_{v}{ }^{(b)} \\
{[\mathrm{Jy}]}\end{array}$ & $\begin{array}{c}d^{(c)} \\
{[\mathrm{mas}]}\end{array}$ & $\begin{array}{c}\phi^{(d)} \\
{\left[{ }^{\circ}\right]}\end{array}$ & $\begin{array}{c}b_{\text {maj }}(e) \\
{[\mathrm{mas}]}\end{array}$ & $\log T_{\mathrm{b}}(f)$ \\
\hline \multicolumn{6}{|c|}{$2007-10-15$ (2007.79) } \\
\hline $\mathrm{C} 1$ & 1.51 & 0.070 & -128.6 & 0.044 & 11.14 \\
\hline $\mathrm{C} 2 \mathrm{a}$ & 2.47 & 0.015 & -138.1 & 0.037 & 11.50 \\
\hline $\mathrm{C} 2 \mathrm{~b}$ & 1.80 & 0.039 & 42.9 & 0.046 & 11.18 \\
\hline $\mathrm{J} 3$ & 1.87 & 0.089 & 67.6 & 0.081 & 10.70 \\
\hline $\mathrm{J} 2 \mathrm{a}$ & 1.77 & 0.256 & 75.8 & 0.067 & 10.84 \\
\hline $\mathrm{J} 2 \mathrm{~b}$ & 1.09 & 0.359 & 85.7 & 0.074 & 10.54 \\
\hline $\mathrm{J} 1$ & 1.53 & 0.590 & 76.8 & 0.120 & 10.27 \\
\hline \multicolumn{6}{|c|}{$2008-05-11(2008.36)$} \\
\hline $\mathrm{C} 1$ & 0.47 & 0.104 & -117.9 & 0.049 & 10.54 \\
\hline $\mathrm{C} 2$ & 1.16 & 0.003 & -145.9 & 0.049 & 10.94 \\
\hline \multirow[t]{2}{*}{ J5 } & 0.55 & 0.089 & 59.4 & 0.089 & 10.08 \\
\hline & 0.33 & 0.357 & 59.9 & 0.150 & 9.41 \\
\hline $\mathrm{J} 4$ & 0.26 & 0.516 & 57.4 & 0.059 & 10.12 \\
\hline $\mathrm{J} 3$ & 0.88 & 0.778 & 64.9 & 0.152 & 9.82 \\
\hline $\mathrm{J} 2$ & 0.52 & 1.003 & 77.6 & 0.197 & 9.37 \\
\hline \multirow[t]{2}{*}{$\mathrm{J} 1$} & 0.14 & 1.297 & 67.4 & 0.250 & 8.61 \\
\hline & 0.06 & 4.108 & 65.9 & 0.166 & 8.60 \\
\hline \multicolumn{6}{|c|}{$2008-10-14(2008.78)$} \\
\hline $\mathrm{C} 1$ & $0.66^{(g)}$ & 0.101 & -117.1 & 0.039 & 10.88 \\
\hline $\mathrm{C} 2$ & $0.74^{(g)}$ & 0.030 & -142.4 & 0.037 & 10.98 \\
\hline \multirow[t]{3}{*}{$\mathrm{C} 2$} & $1.44^{(g)}$ & 0.009 & 33.6 & 0.024 & 11.65 \\
\hline & $0.38^{(g)}$ & 0.059 & 53.5 & 0.061 & 10.26 \\
\hline & $0.11^{(g)}$ & 0.385 & 57.1 & 0.148 & 8.96 \\
\hline $\mathrm{J} 5$ & $0.10^{(g)}$ & 0.680 & 59.4 & 0.107 & 9.18 \\
\hline $\mathrm{J} 4$ & $0.06^{(g)}$ & 1.099 & 64.8 & 0.082 & 9.19 \\
\hline $\mathrm{J} 3$ & $0.10^{(g)}$ & 1.418 & 63.8 & 0.110 & 9.14 \\
\hline $\mathrm{J} 2$ & $0.17^{(g)}$ & 1.509 & 76.5 & 0.306 & 8.51 \\
\hline \multirow{2}{*}{$\mathrm{J} 1$} & $0.05^{(g)}$ & 1.750 & 65.4 & 0.201 & 8.31 \\
\hline & $0.06^{(g)}$ & 4.739 & 66.1 & 0.496 & 7.60 \\
\hline
\end{tabular}

Notes. ${ }^{(a)}$ ID of kinematic component. ${ }^{(b)}$ Flux density of the component. ${ }^{(c)}$ Distance of the component to center of the map. ${ }^{(d)}$ Position angle of the component relative to the center of the map. ${ }^{(e)}$ Major axis of the Gaussian component. ${ }^{(f)}$ Logarithmic value of the brightness Temperature. ${ }^{(g)}$ As noted in Sect. 2.1, the flux density values obtained for the third VLBI measuremnt might be systematically underestimated by $22 \%$.
Table A.3. Coordinates of components at $15 \mathrm{GHz}$ (shift applied), $43 \mathrm{GHz}$, and $86 \mathrm{GHz}$ used for Fig. 5.

\begin{tabular}{lccc}
\hline \hline ID $^{(a)}$ & $\begin{array}{c}v^{(b)} \\
{[\mathrm{GHz}]}\end{array}$ & $\begin{array}{c}\text { Rel. RA } \\
{[\mathrm{mas}]}\end{array}$ & $\begin{array}{c}\text { Rel. Dec }^{(d)} \\
{[\mathrm{mas}]}\end{array}$ \\
\hline B1 & 15 & 1.51 & 0.65 \\
& 43 & 1.56 & 0.75 \\
$\mathrm{~J} 1$ & 86 & 1.68 & 0.78 \\
\hline B4 & 15 & 1.29 & 0.20 \\
& 43 & 1.41 & 0.37 \\
$\mathrm{~J} 2$ & 86 & 1.56 & 0.40 \\
\hline B2 & 15 & 1.23 & 0.47 \\
& 43 & 1.20 & 0.57 \\
$\mathrm{~J} 3$ & 86 & 1.36 & 0.67 \\
\hline B3 & 15 & 0.93 & 0.40 \\
& 43 & 0.84 & 0.42 \\
$\mathrm{~J} 4$ & 86 & 1.08 & 0.51 \\
\hline
\end{tabular}

Notes. ${ }^{(a)}$ ID of kinematic component. ${ }^{(b)}$ Frequency of the VLBI observation. ${ }^{\left({ }^{c}\right)}$ Relative Right Ascension of the component. ${ }^{(d)}$ Relative Declination of the component. 
Table A.4. $86 \mathrm{GHz}$ flux density measurement from F-GAMMA and POLAMI.

\begin{tabular}{|c|c|c|c|}
\hline $\begin{array}{l}\text { Date }^{(a)} \\
{[\mathrm{MJD}]}\end{array}$ & $\underset{[\mathrm{Jy}]}{\text { Flux }^{(b)}}$ & $\begin{array}{c}\text { Flux error }{ }^{(c)} \\
{[\mathrm{Jy}]}\end{array}$ & Project $(d)$ \\
\hline 54022.014 & 3.92 & 0.2 & POLAMI \\
\hline 54113.504 & 4.11 & 0.07 & F-GAMMA \\
\hline 54229.991 & 7.0 & 0.36 & POLAMI \\
\hline 54236.013 & 7.38 & 0.38 & POLAMI \\
\hline 54261.200 & 10.033 & 0.71 & F-GAMMA \\
\hline 54312.617 & 12.444 & 0.258 & F-GAMMA \\
\hline 54323.149 & 13.374 & 0.988 & F-GAMMA \\
\hline 54332.994 & 13.46 & 0.7 & POLAMI \\
\hline 54357.996 & 10.96 & 0.57 & POLAMI \\
\hline 54359.018 & 12.83 & 0.66 & POLAMI \\
\hline 54360.745 & 12.613 & 0.249 & F-GAMMA \\
\hline 54382.479 & 12.057 & 0.224 & F-GAMMA \\
\hline 54384.021 & 11.6 & 0.6 & POLAMI \\
\hline 54447.249 & 10.482 & 0.51 & F-GAMMA \\
\hline 54479.560 & 7.561 & 0.11 & F-GAMMA \\
\hline 54507.508 & 6.898 & 0.199 & F-GAMMA \\
\hline 54528.401 & 5.806 & 0.08 & F-GAMMA \\
\hline 54588.072 & 4.055 & 0.158 & F-GAMMA \\
\hline 54593.002 & 3.91 & 0.2 & POLAMI \\
\hline 54616.812 & 3.903 & 0.066 & F-GAMMA \\
\hline 54618.000 & 4.03 & 0.21 & POLAMI \\
\hline 54627.992 & 4.08 & 0.21 & POLAMI \\
\hline 54636.995 & 3.65 & 0.19 & POLAMI \\
\hline 54642.977 & 3.373 & 0.206 & F-GAMMA \\
\hline 54649.000 & 3.57 & 0.18 & POLAMI \\
\hline 54675.730 & 3.619 & 0.137 & F-GAMMA \\
\hline 54703.592 & 2.888 & 0.16 & F-GAMMA \\
\hline 54720.004 & 3.21 & 0.17 & POLAMI \\
\hline 54724.800 & 3.718 & 0.148 & F-GAMMA \\
\hline 54746.537 & 4.061 & 0.215 & F-GAMMA \\
\hline 54747.015 & 4.37 & 0.23 & POLAMI \\
\hline 54777.355 & 6.71 & 0.757 & F-GAMMA \\
\hline 54806.356 & 5.135 & 0.219 & F-GAMMA \\
\hline 54830.024 & 4.02 & 0.21 & POLAMI \\
\hline 54837.555 & 4.363 & 0.171 & F-GAMMA \\
\hline 54882.005 & 3.84 & 0.2 & POLAMI \\
\hline 54898.013 & 3.453 & 0.114 & F-GAMMA \\
\hline 54963.848 & 2.171 & 0.109 & F-GAMMA \\
\hline 55004.778 & 2.135 & 0.196 & F-GAMMA \\
\hline
\end{tabular}

Notes. ${ }^{(a)}$ Date of flux density measurement. ${ }^{(b)}$ Flux density measurement. ${ }^{(c)}$ Uncertainty of flux density measurement. ${ }^{(d)}$ Flux density monitoring programme which obtained the measurement. 